\title{
ELECTRON LIQUIDS IN COUPLED QUANTUM WELLS
}

\author{
L. ŚWIERKoWSKI ${ }^{a}$, J. SzYMAŃSKI $^{b}$ AND D. NeILSON ${ }^{a}$ \\ a School of Physics, University of New South Wales, Kensington 2033, Australia \\ ${ }^{b}$ Telecom Research Laboratories, 770 Blackburn Road, Clayton Vic. 3168, Australia
}

\begin{abstract}
A system comprising of two adjacent layers of conduction electrons or alternatively a layer of electrons and a layer of holes can exhibit novel instabilities in the liquid phase towards inhomogeneous ground states. The carriers in the two layers can couple to each other through the Coulomb interaction but they are not permitted to tunnel so that the charges in one layer act as a polarisable background for the other layer. The presence of a second layer encourages the formation of novel ground states with inhomogeneous density distributions. We find theoretical evidence for the existence of charge density wave ground states and also a coupled Wigner crystal. These exist at much higher densitics than the Wigner crystallisation density for the single layer case. The existence of these inhomogeneous ground states leads to significant modifications of the low lying excitation spectrum in the uniform liquid phase. Near the transitions to both the charge density wave and the coupled Wigner crystal phases we find evidence of the development in the liquid phase of new soft mode excitations of finite wave number $\boldsymbol{q}$ that are precursors of the inhomogeneous ground states. Near the transition to the coupled Wigner crystal we observe a strong tendency of the single particle excitation spectrum for the liquid phase to renormalise into a single line that has a dispersion closely resembling the phonon dispersion curve for the solid.
\end{abstract}

PACS numbers: 73.40.Lq, 71.45.Gm, 71.45.Lr

\section{Introduction}

In ordinary metals the conduction electrons are only weakly interacting, the potential energy playing only a minor role and interaction effects being well accounted for by linear screening. The excitation spectrum of the system consists only of non-interacting single-particle excitations together with the plasmon collective mode.

At very low densities Coulomb correlations become the dominant effect and they can control both the type of ground state that is formed and the nature of the excitation spectrum. Thus, for example, in the pure electron solid the low 
lying excitation spectrum contains phonons rather than plasmons and it is an interesting question to consider the extent to which the change in the spectrum from the plasmon collective mode plus single-particle excitations of the liquid across to the phonon line of the solid is a continuous evolution or a sudden jump. In conventional materials the densities needed to access the strongly correlated region of phase space for the electrons are so small that experimental observations within this region have only been possible in strong external magnetic fields. The lowest density of electrons in bulk metals corresponds to about $r_{s}=6$ whereas the transition to the electron solid (the Wigner crystal) in zero magnetic field is predicted to occur close to $r_{s}=100$ [1]. The paramter $r_{s}$ is the average spacing between electrons in units of the effective Bohr radius $a_{\mathrm{B}}^{*}$.

During the past decade there has been a great deal of interest in the high-mobility two-dimensional electron system. Electrons trapped in thin layers at interfaces of gallium arsenide heterostructures are more strongly correlated than electrons in the bulk at the same density because of the smaller kinetic energy contribution in a system with only two spatial degrees of freedom. The Wigner crystal transition in two dimensions for example is predicted to occur at $r_{s}=37 \pm 5$ [2]. IIowever the small electron eflective mass in GaAs, $m^{*} / m \approx 0.07$, and the large dielectric constant which for GaAs is $\varepsilon \approx 13$ means that in $r_{s}$ terms even the lowest density of electrons in a conducting layer is in the relatively high density region $r_{s} \leq 5$. IIoles have larger effective masses and densities as low as $r_{s}=19$ have recently been reported for layers of holes [3].

We have proposed that strong correlations in the eleciron or hole liquid could be further investigated cxperimentally in the absence of a magnetic field by using semiconductor heterostructures with two coupled layers $[4,5]$ of charge carriers. The correlations in two layer systems are stronger than for a single layer because the charge carriers in the first laycr can couple to the carriers in the second layer and vice versa, so that each layer acts as a polarisable background for the other. A continuous range of densities in one of the layers can be obtained by using a gated structure.

We have shown that the two-layer system has a rich diagram of inhomogeneous ground states, including Wigner crystals and charge density waves (CDW) [4]. Charge density waves can occur at densities much higher than those at which the transition into the Wigner crystal occurs.

The mixed system of one layer of electrons and the other of holes may be an even richer subject for study from both theoretical and experimental viewpoints. The interaction betwcen layers is attractive, the attractive interlayer correlations competing with the rejulsive correlations within each layer. The electrons and holes can pair into excil onic-like bound states leading to additional exotic ground states including the possibility of superfluidity $[6,7]$.

\section{Response function of the system}

The system we are considering consists either of two layers of electrons or one layer of electrons and one layer of holes trapped at two adjacent interfaces in a semiconductor heterostructure. We assume that only the lowest subband in the 
potential well at the interface is occupied. In the following formalism all interactions contain suitable form factors to account for the finite width of the layers. If an external potential $V_{l}^{\text {ext }}(q, \omega)$ is applied to the system it will induce density changes $\delta n_{1}$ and $\delta n_{2}$ in the two layers. The total potential acting on the first layer will be $V_{1}^{\text {ext }}(q, \omega)$ plus the interaction induced by changes in electron density in the second layer $\delta n_{2}$, and vice versa. The induced electron density in the $l$ th layer will be

$$
\delta n_{l}(\boldsymbol{q}, \omega)=-\chi^{s}(\boldsymbol{q}, \omega)\left[V_{l}^{\mathrm{ext}}(\boldsymbol{q}, \omega)+\sum_{l^{\prime}=1}^{2}\left[1-G_{l l^{\prime}}(\boldsymbol{q})\right] V_{l l^{\prime}}(\boldsymbol{q}) \delta n_{l^{\prime}}(\boldsymbol{q}, \omega)\right],
$$

where $V_{l \prime}(q)$ is the bare Coulomb intercation between electrons either in the same layer $\left(l=l^{\prime}\right)$ or in different layers $\left(l \neq l^{\prime}\right)$. The response function of the system $\chi^{s}(q, \omega)$ neglects the interaction between the charged carriers but takes into account the effect of scattering by defects

$$
\chi^{s}(q, \omega)=\frac{\chi^{0}\left(q, \omega-\gamma^{s}\right)}{1+\frac{\gamma^{s}}{\omega-\gamma^{s}}\left[1-\frac{\chi^{0}\left(q, \omega-\gamma^{s}\right)}{\chi^{0}(q)}\right]},
$$

$\chi^{0}(q, \omega)$ is the finite temperature Lindhard function. The single-particle memory function $\gamma^{s}(\boldsymbol{q}, \omega)$ [8] accounts for scattering of the carriers by defects. Here we set $\gamma^{s}(q, \omega)$ equal to a constant, $\gamma^{s}=(\mathrm{i} \tau)^{-1}$, where $\tau$ represents the time between scattering of a charged carrier off defects. $\tau$ is related to the electron mobility parallel to the layer $\mu$ by the usual Drude expression.

The static local fields [9] $G_{n \prime}(q)$ in Eq. (1) reduce the strength of the interaction between two electrons because of the reduction in the probability of finding an electron in the vicinity of another electron.

The response function matrix of the two layer system $\chi_{m}(\boldsymbol{q}, \omega)$ is defined by

$$
\delta n_{l}(\boldsymbol{q}, \omega)=-\sum_{l^{\prime}=1}^{2} \chi_{u^{\prime}}(\boldsymbol{q}, \omega) V_{l^{\prime}}^{\mathrm{ext}}(\boldsymbol{q}, \omega) .
$$

The matrix $\chi u^{\prime}(q, \omega)$ can be diagonalised, the diagonal elements $\chi_{ \pm}(q, \omega)$ describing the response of the system in which the density modulations $\delta n_{l}(\boldsymbol{q}, \omega)$ in the two layers are in phase for the + label and $\pi$ out of phase for the - label. Using Eqs. (1) and (3) we obtain

$$
\chi_{ \pm}(q, \omega)=\frac{\chi^{s}(q, \omega)}{1+\left\{V_{11}(q)\left[1-G_{11}(q)\right] \pm V_{12}(q)\left[1-G_{12}(q]\right\} \chi^{s}(q, \omega)\right.} .
$$

The static local field $G_{11}(q)$ within a layer is extracted in our approach from the Monte Carlo simulations results using the procedure introduced by Świerkowski et al. [4] and Neilson et al. [8]. First we write the response function for a single isolated layer $\chi(\boldsymbol{q}, \omega)$, i.e. in the absence of coupling between layers,

$$
\chi(q, \omega)=\frac{\chi^{s}(q, \omega)}{1+V_{11}(q)\left[1-G_{11}(q)\right] \chi^{s}(q, \omega)} .
$$

Tanatar and Ceperley [2] calculated the pair correlation function $g(r)$ for the range of densities down to the crystallisation point using Monte Carlo methods. The Fourier transform of the pair correlation function gives the static structure factor 
which in turn is linked with the local field in Eq. (5) by the fluctuation-dissipation theorem. It is worth noting at this point that the pair correlation function $g_{11}(r)$ obtained from Eq. (7) in the presence of interlayer interactions is non identical to the Monte Carlo $g(\boldsymbol{r})$.

Since in the absence of tunnelling the two-layer system is formally equivalent to a two-component plasma, we can calculate the interlayer local field $G_{12}(q)$ using the method introduced by Singwi, Tosi, Land and Sjölander (STLS) [10] generalised to a two-component plasma [11]. In the STLS approach the density-density correlation function is approximated by a product of two densities times the static pair correlation function,

$$
\left\langle\delta \widehat{n}_{1}(r, t) \delta \widehat{n}_{2}\left(r^{\prime}, t\right)\right\rangle \approx \delta n_{1}(r, t) g_{12}\left(r-r^{\prime}\right) \delta n_{2}\left(r^{\prime}, t\right) .
$$

The symbol ${ }^{\wedge}$ distinguishes the operators $\delta \widehat{n}_{l}(r, t)$ from their expectation values $\delta n_{l}(r, t)$. If we replaced $g_{12}\left(r-r^{\prime}\right)$ in Eq. (6) by unity we would recover the IIartree expression.

Equation (6), together with the relation linking the pair correlation function $g_{l^{\prime}}(r)$ with the static structure factor $S_{l l^{\prime}}(q)$,

$$
g_{l \prime}(r)=1+\frac{1}{n} \int \frac{\mathrm{d}^{2} q}{(2 \pi)^{2}} \mathrm{e}^{\mathrm{i} \boldsymbol{q} \cdot \boldsymbol{r}}\left[S_{l^{\prime}}(q)-\delta_{l l^{\prime}}\right],
$$

gives an axpression for the local field [10-12],

$$
G_{12}(q)=-\frac{1}{n} \int \frac{\mathrm{d}^{2} k}{(2 \pi)^{2}} \frac{(q \cdot k)}{q^{2}} \frac{V_{12}(k)}{V_{12}(q)} S_{12}(|q-k|) .
$$

$n$ is the average density in one layer. Equations (4) and (8) taken with the fluctuation-dissipation theorem

$$
S_{u^{\prime}}(q)=\frac{\hbar}{\pi n} \int_{0}^{\infty} \mathrm{d} \omega \frac{\operatorname{Im} \chi_{u^{\prime}}(q, \omega)}{1-\exp \left(-\hbar \omega / k_{\mathrm{B}} T\right)},
$$

make up a set of self-consistent equations for $G_{12}(q)$ [13]. For simplicity we have assumed in Eqs. (4-9) that the densities in both layers are identical. The generalisation to the case of different densities is straightforward.

The procedure for calculating $G_{11}(q)$ and $G_{22}(q)$ from the Monte Carlo results for uncoupled layers and calculating $G_{12}(q)$ using STLS can only be fully justified in the case of weak coupling between layers. In other cases (which would include some of our low density results) this approach should be regarded as an initial approximation.

\section{Ground state properties}

We have used the theory developed in the previous section to calculate the static response functions $\chi_{ \pm}(q)$ at a number of different interlayer spacings $a$ for a fixed density. We find if the layer spacing is decreased sufficiently that $\chi_{-}(q)$ can develop a singularity at some finite $q$.

Figure 1 shows the static diagonal element $\chi_{-}(q)$ for two layers of electrons. For $\chi_{-}(q)$ the density modulations $\delta n_{l}$ in the adjacent layers are $\pi$ out-of-phase. This configuration has a lower energy than the configuration in which the density 
modulations for the two-electron layers are in-phase. Figure 1 shows results for two density values, $r_{s}=10$ and 30 for a number of interlayer spacings $a$.

For $r_{s}=10$ the peak in $\chi_{-}(q)$ is centred at $|q|=2 k_{\mathrm{F}}$. As $a$ is decreased the peak grows and finally diverges when $a=a_{\mathrm{c}} \approx 37 \mathrm{~nm}$.

For $r_{s}=30, a_{c} \approx 195 \mathrm{~nm}$. There are two peaks in $\chi_{-}(q)$, one centred at $|q|=2 k_{F}$ and other at a somewhat larger value of $|q|$. We interpret the peak at $|q|=2 k_{\mathrm{F}}$ as an incipient instability to a charge density wave ground state. The peak at $|q| \approx 2.5 k_{F}$ we associate with an incipient instability to coupled Wigner lattices in the two layers. As the layers are brought closer together it is the charge density wave peak that eventually diverges. We find for densities below $r_{s}=30$ the Wigner crystal peak becomes the first of the two peaks to diverge.

The divergence of the static response function establishes some limits on the stability of the liquid phases in the two layers. Figure 2a shows a possible phase diagram for two-electron layers as a function of the electron density in each layer $r_{s}$ and the spacing between the layers $a$. Three phase regions are shown, the liquid state, the charge density wave (CDW) ground state and the Wigner crystal. For fixed $r_{s}$ in the range $5 \leq r_{s} \leq 30$, there is a transition as the layer spacing is decreased from the liquid to a charge density wave ground state. For $r_{s}$ in the range $30 \leq r_{s} \leq 37$, the transition from the liquid is into a Wigner crystal state. Monte-Carlo data tells us that for $r_{s} \geq 37$ the ground state for the single layer is the Wigner crystal [2]. The single layer corresponds in our formalism to the limit when the two layers are not coupled, $a \rightarrow \infty$. For very small $a$ the real system should once again resemble a single layer as the two layers merge and we speculate there is another phase transition back to the liquid ground state as a goes to zero.

For a layer of electrons coupling to a layer of holes it is the in-phase response function $\chi_{+}(q)$ that can become singular. Figure $2 \mathrm{~b}$ shows the phase diagram for this case. This system would be expected to crystallise more readily due to the attractive interaction between the electrons and the holes, and we find indeed that the transition to the Wigner crystal occurs at a density as high as $r_{s}=15$ for a
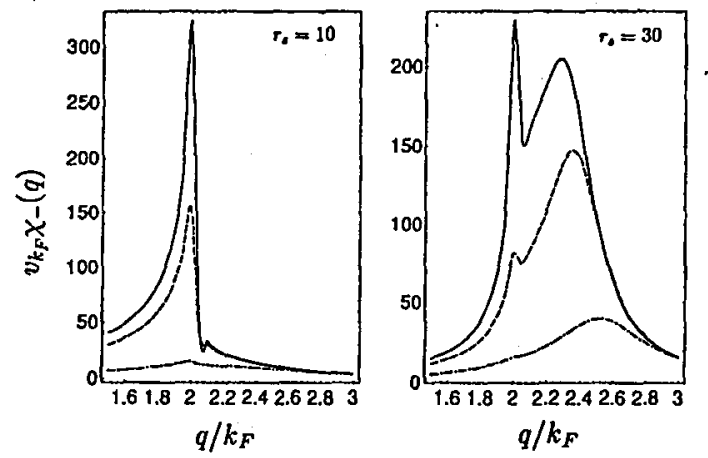

Fig. 1. The static response function $\chi_{-}(q)$ for densities $r_{s}=10$ and 30 and $a=2 a_{c}$ (chain line), $a=1.1 a_{c}$ (broken line) and $a=1.01 a_{c}$ (solid line). The factor $v_{k_{F}}=$ $2 \pi e^{2} / k_{\mathrm{F}}$. 

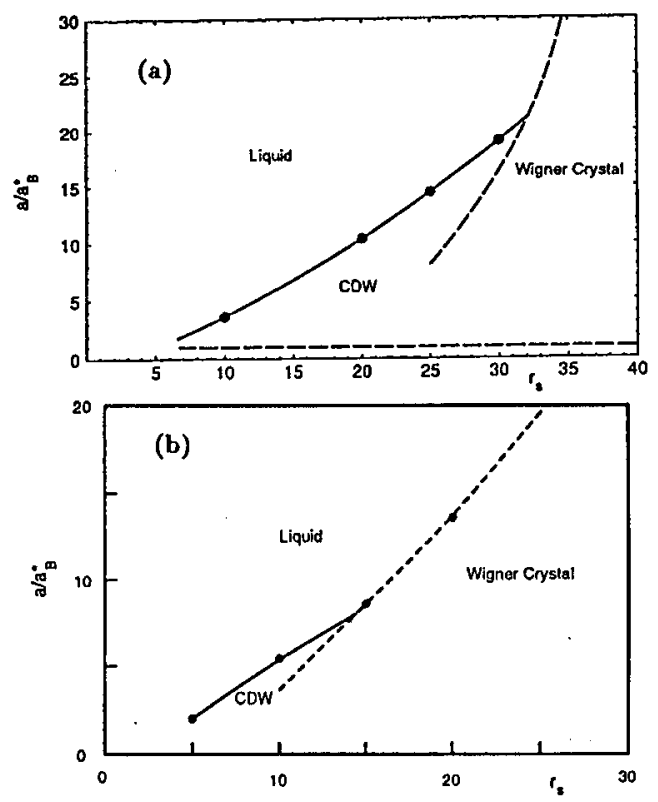

Fig. 2. a) Possible phase diagram for two-electron layers, as a function of the electron density $r_{s}$ and the layer spacing $a$. The phases are the liquid state, the charge density wave ground state CDW and the Wigner crystal. b) Possible phase diagram for the two-layer electron-hole system.

layer spacing of $a_{\mathrm{c}} \approx 80 \mathrm{~nm}$. At higher densities the transition is to a charge density wave, one in which the density modulations in the two layers will be in-phase. For coupled electron-hole layers the situations is complicated as the layers are moved together and there are a variety of possible ground states of the system $[6,7]$. This is beyond the scope of the model we are using and we do not attempt to speculate on the nature of the phase diagram for small $a$.

It is instructive to show the behaviour of the pair correlation functions close to a transition. Once the local fields are known, Eqs. (4), (7) and (9) can be used to obtain the pair correlation functions. Figure 3 shows the intralayer correlation function $g_{11}(r)$ and the interlayer correlation function $g_{12}(r)$ for coupled electron layers at a density of $r_{s}=30$ for the interlayer spacing, $a=1.01 a_{\mathrm{c}} \approx 195 \mathrm{~nm}$. Very close to the transition the oscillatory peaks in $g_{11}(r)$ and $g_{12}(r)$ become pronounced. This is to be expected and reflects the proximity in energy of an excited state with an inhomogeneous density distribution. The matching of maxima of $g_{11}(r)$ with the minima of $g_{12}(r)$ can clearly be seen and indicate the extent to which the correlations in the two layers act to strongly compensate each other.

Figure 4 shows the corresponding results for coupled electron-hole layers with $a=1.01 a_{\mathrm{c}} \approx 135 \mathrm{~nm} . g_{12}(r)$ (broken line) is greater than unity for small $r$ 
because of the attractive interaction. Within the STLS approximation formation of a bound exciton pair would show up as a divergence of the calculated $g_{12}(r)$ for small $r$ [14].

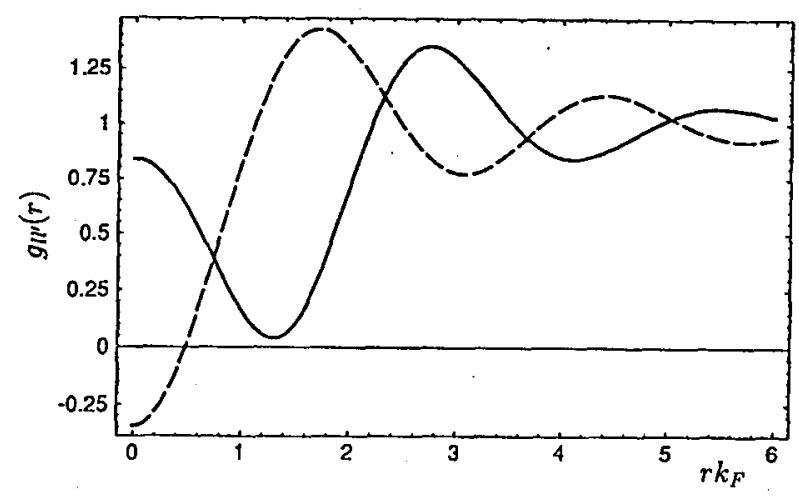

Fig. 3. The intralayer correlation function $g_{11}(r)$ (full line) and the interlayer correlation function $g_{12}(r)$ (broken line) for two coupled electron layers at a density of $r_{s}=30$ for an interlayer spacing of $a=1.01 a_{c}$.

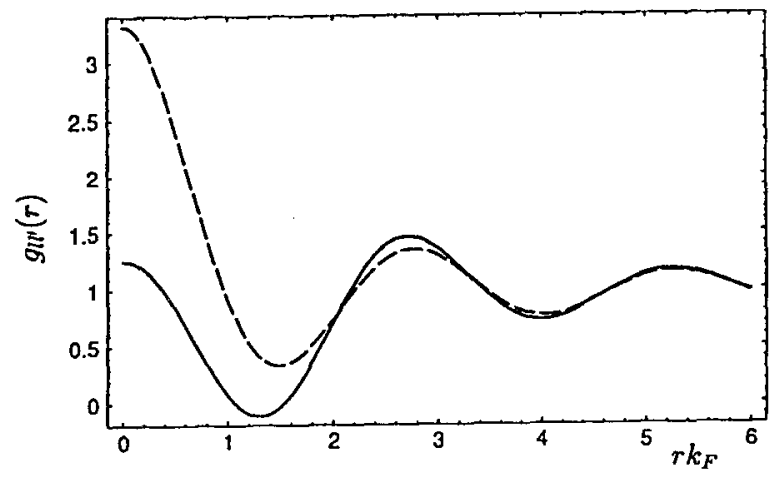

Fig. 4. The intralayer correlation function $g_{11}(r)$ (full line) and the interlayer correlation function $g_{12}(\boldsymbol{r})$ (broken line) for a layer of electrons coupled to a layer of holes, each at a density of $r_{s}=20$ for an interlayer spacing of $a=1.01 a_{\mathrm{c}}$.

\section{Excitation spectrum of the two-layer system}

It is well known from random phase approximation (RPA) calculations that the collective mode spectrum of the many layer systems is much richer than that of a single layer. The plasmon mode for the $N$-layer case splits into $N$ collective 
excitations, and for an infinite number of layers (as superlattice) a plasmon band is formed.

We find that correlations are responsible not only for quantitative changes in the collective mode spectrum but that the changes can affect the nature of the spectrum itself. Modes can disappear altogether, while new modes, associated with the phase transition from liquid to more ordered phases can appear.

\subsection{Plasmon modes}

Figure 5 shows the low lying collective modes for two coupled layers for a spacing between the layers of $a=47 \mathrm{~nm}$ compared with RPA [15]. One of the two

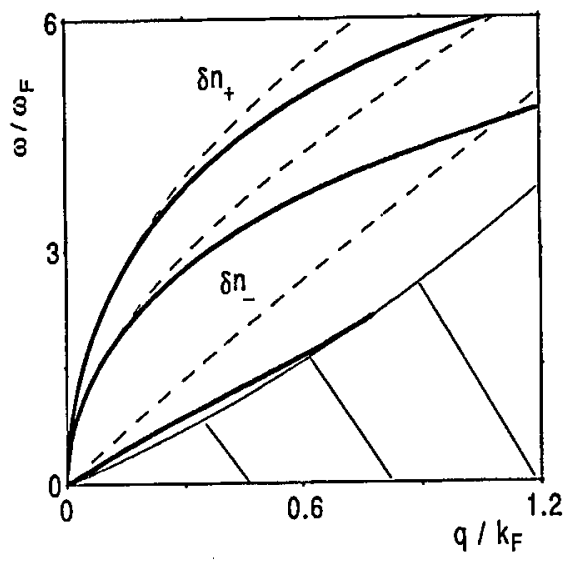

Fig. 5. Dispersion curves for the collective modes for two layers for $r_{s}=\mathbf{1 0}$ and layer spacing $a=47 \mathrm{~nm}$. The solid lines which include many-body effects are the dispersion curves for the eigenmodes $\delta n_{-}(q, \omega)$ and $\delta n_{+}(q, \omega)$ for the two-layer system. The central solid dispersion curve refers to the plasmon eigenmode for a single layer (see Neilson et al. [8]). The dotted lines show the corresponding modes calculated within RPA. Note the significant effect of many-body correlations on the long wave-length dispersion of the $\delta n_{-}(q, \omega)$ mode.

collective modes has a plasmon-like dispersion as $q \rightarrow 0\left(\hbar \omega \approx|q|^{1 / 2}\right)$ while the energy of the second mode ("acoustic" plasmon) vanishes linearly with $q$ because the out of phase density modulations in the opposite layer screen out the long range part of the Coulomb potential.

It is interesting to contrast how many-body correlations between electrons affect the long wave-length dispersion of these two eigenmodes. Their inclusion through the local fields $G_{l \prime}(q)$ decreases the effective strength of the Coulomb interaction so the gradients of both plasmon dispersion curves are less than for RPA. We see in Fig. 5 that it is only at relatively large wave numbers that many-body correlations affect the plasmon for the single layer and the plasmon corresponding to the $\delta n_{+}(q, \omega)$ eigenmode. This is to be expected since many-body correlations primarily affect properties of the electrons at small separations. However the result 
for the acoustic plasmon corresponding to the $\delta n_{-}(q, \omega)$ eigenmode is noteworthy. Figure 5 shows that many-body correlations affect this plasmon down to essentially zero wave number. Our calculations for smaller layer spacings show that many-body correlations may push the RPA acoustic plasmon almost completely into the single-particle excitation region and it ceases to exist as an independent collective excitation.

\subsection{CDW instability}

At $r_{s}=10$ the instability towards a charge density wave occurs when the spacing between the layers has a value $a_{\mathrm{c}}=43.5 \mathrm{~nm}$. Figure 6 shows $\operatorname{Im} \chi_{-}(q, \omega)$ at fixed $q=2 k_{\mathrm{F}}$. There is a strong shift of spectral strength within the single

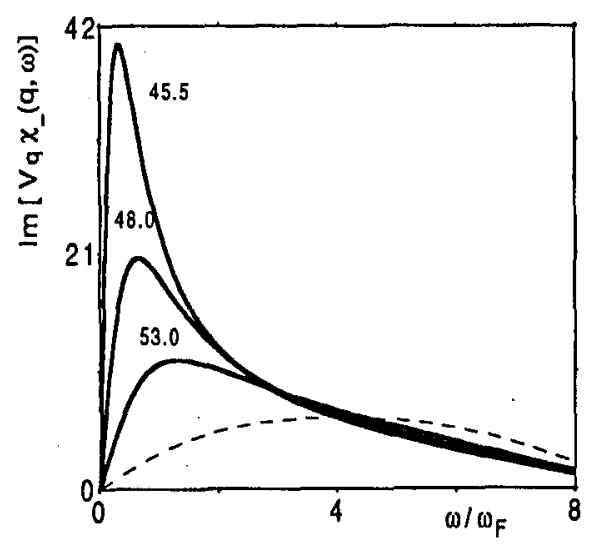

Fig. 6. $\operatorname{Im} \chi-(q, \omega)$ at $q=2 k_{\mathrm{F}}$ and $r_{s}=10$ as a function of the spacing between the layers $a$, with the numbers given in units of $\mathrm{nm}$. The instability occurs at $a=43.5 \mathrm{~nm}$. The temperature has been taken to be $500 \mathrm{mK}$ and the electron mobility of the sample as $\mu=10^{5} \mathrm{~V} \mathrm{~cm}^{-2}$.

particle excitation region towards momentum transfer $q \approx 2 k_{\mathrm{F}}$. This indicates a concentration of low lying excited states with a density modulation period close to that of the incipient charge density wave instability. The appearance of this mode indicates that it costs relatively little energy to excite the system into a state with a periodic modulation of the density. The closer the soft mode is to zero energy the longer spontaneous fluctuations into a density modulated excited state can remain and there would be a tendency for the system to be unstable to a periodic charge density wave ground state.

As the precursor of the charge density wave ground state in the liquid phase the soft mode could be used to experimentally confirm the existence of charge density waves. A sizeable new peak appearing in the liquid structure factor $S(q, \omega)$ at $q \approx 2 k_{\mathrm{F}}$ and small $\omega$ would be observable using standard Raman scattering techniques. 


\subsection{Wigner crystal instability}

For some interlayer distances at low density a new mode appears on the large $q$, low energy side of the single-particle region of $\operatorname{Im} \chi_{-}(q, \omega)$ (Fig. 7). This new

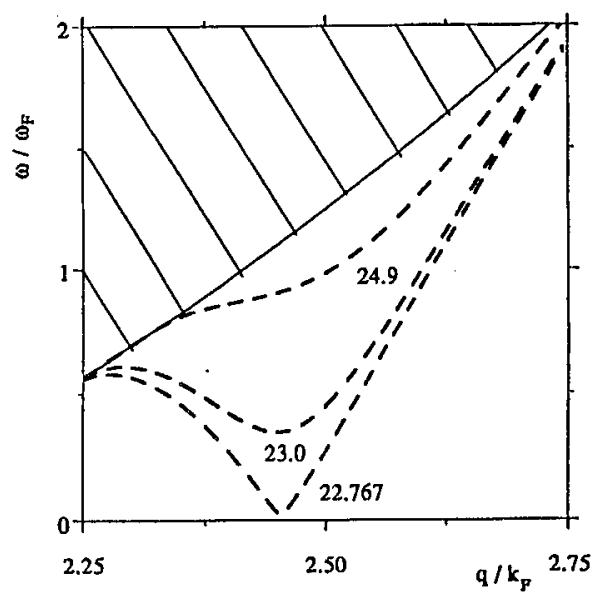

Fig. 7. Dispersion curves for new large $q$ collective mode at $r_{s}=32$. The labels refer to the spacing betwcen layers in units of the effective Bohr radius ( $a_{\mathrm{B}}^{*}=9.8 \mathrm{~nm}$ for $\mathrm{GaAs}$ ).

mode occurs only for a narrow range of $q$ centred on $|q|=|G| \approx 2.45 k_{F}$, where $G$ is the primitive reciprocal lattice vector in the $X$ direction for a hexagonal Wigner lattice at the same density. The energy of this mode is very small and if the layers are moved closer together, the energy of the mode drops towards zero. We interpret this mode as a quasi soft mode previously reported for the single layer [8], a dynamic precursor to the Wigner phase. This precursor is a low lying transient excitation of the liquid phase which mimics the symmetry of the Wigner lattic. At the phase transition from the liquid to the solid the mode touches $\omega=0$ precisely at $\boldsymbol{q}=\boldsymbol{G}$. The softening of the mode is correlated with the enhancement of the static response function that occurs in the vicinity of $q=G$. When the mode softens to zero energy the static response function becomes singular for $q=0$.

An overall view of the spectral strength of the excitations, $\operatorname{Im} \chi_{-}(q, \omega)$, for $r_{s}=32$ as a function of $q$ and $\omega$ is given in Fig. 8. On the high energy side of the single-particle excitation region we see the plasmon. For clarity it is represented by a series of discrete peaks and we have assumed here a small value for $\gamma_{s}$. On the high- $q$, small $\omega$ side of the single-particle excitation region the soft-mode is clearly visible. It is also clear that there is a collapse of single-particle spectral strength into a distinct ridge which connects the plasmon cut-off on one side with the new collective mode on the other.

We interpret this as showing that the spectral strength of the liquid is evolving into a single dispersion curve with a shape similar to that of the phonon in the Wigner crystal. We have previously seen evidence for this with the solidification transition for a single layer [16]. Figure 9 taken from Ref. [16] shows the phonon 


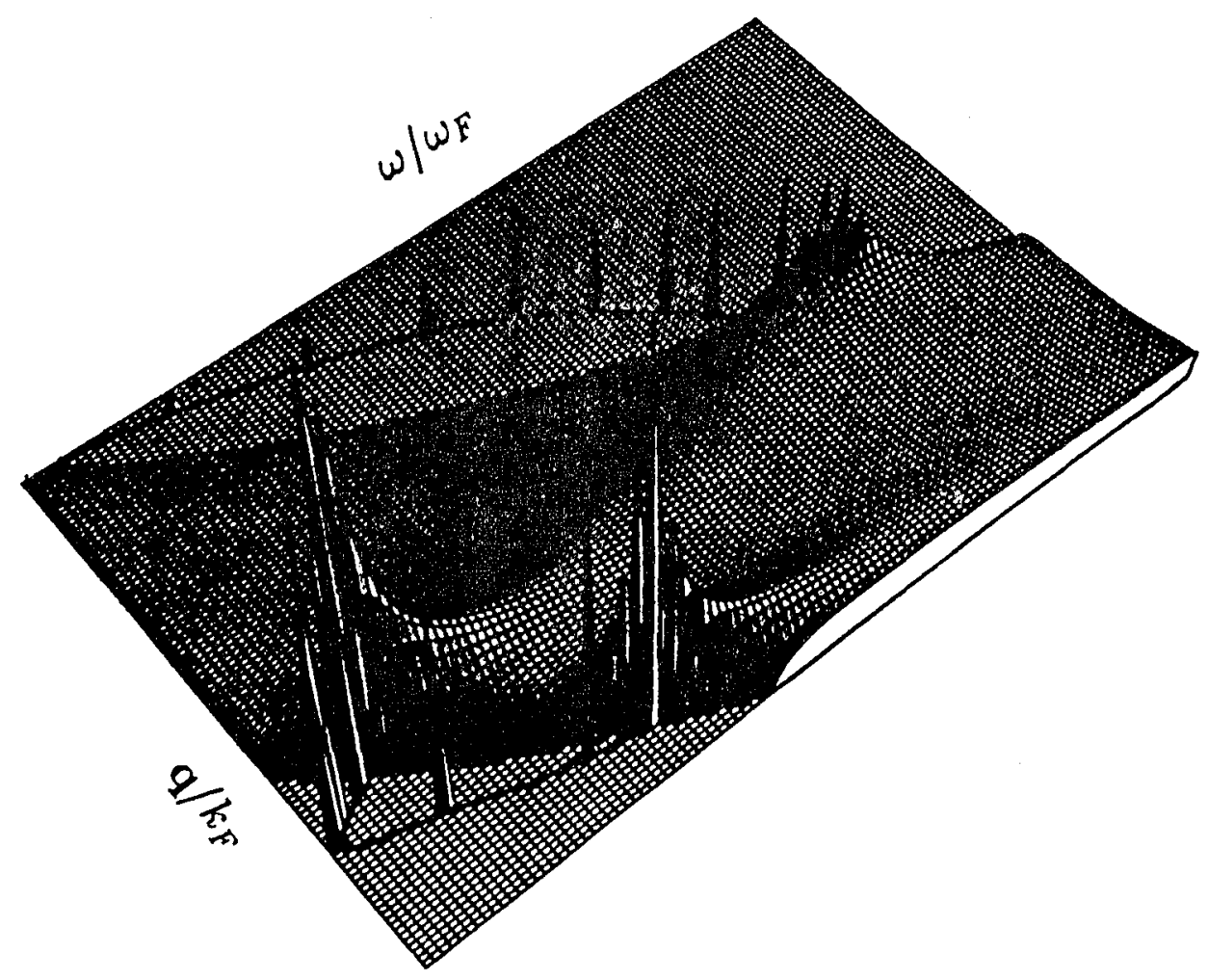

Fig. 8. $\operatorname{Im} \chi-(q, \omega)$ for $r_{s}=32$ as a function of $q$ and $\omega$.

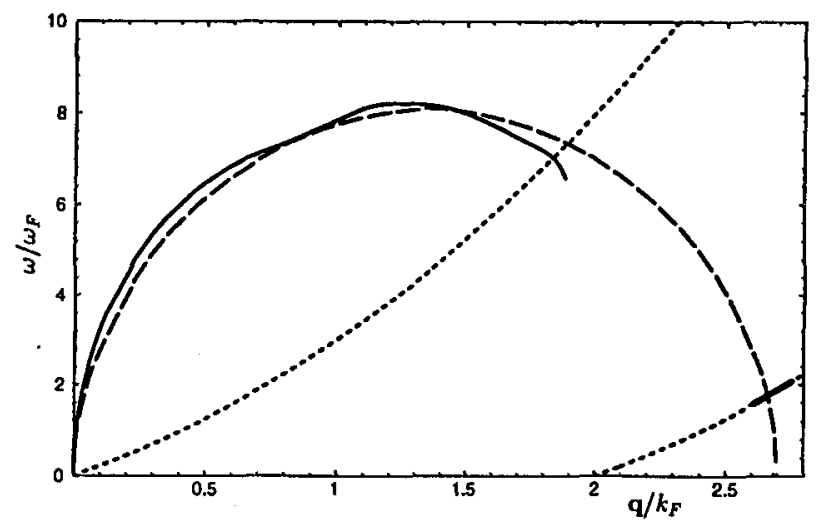

Fig. 9. Plasmon dispersion curve for a single electron layer in the liquid phase (solid line) and the phonon curve (dashed line) of the Wigner phase in the $X$ direction of the Brillouin zone. In both cases $r_{s}=35$. The dotted boundary is the edge of the RPA single-particle excitation region for the liquid. 
dispersion curve for a single layer at density $r_{s}=35$ taken from Bonsall and Maradudin [17]. In two dimensions the lattice configuration with the lowest energy has hexagonal symmetry. Figure 9 is in the $X$ direction of the Brillouin zone. The curve has an axis of symmetry at $g=\frac{1}{2} G$. Figure 9 compares the phonon curve of the solid with the full many-body plasmon dispersion curve for the liquid at the same density calculated by Neilson et al. [8]. The negative dispersion of the plasmon curve is caused by dynamic correlations electrons in the liquid. For small $|q| \ll k_{F}$ the plasmon and phonon curves must agree because of the long range nature of the Coulomb interaction, but for values of $|\boldsymbol{q}|$ comparable to the inverse of the interparticle spacing in the liquid there is no axis of symmetry and no a priori reason to expect the plasmon to bend over at the same rate as the phonon. The new low energy collective mode in the liquid phase matches up with the phonon curve near $|q|=|G|$, so the dynamic correlations in the liquid phase also reflect the hexagonal symmetry of the two-dimensional solid.

\section{Conclusions}

The properties of two-layer systems are markedly different from the properties of electrons in a single layer. Interactions become much more important at relatively higher densitics and the strong correlations can fundamentally change the nature of the ground state. We found for layer densities as high as $r_{s}=5$ that a charge density wave ground state can develop if the layers are sufficiently close to each other. For a layer of electrons coupled to a layer of holes a coupled Wigner crystal ground state can form for carrier densities of $r_{s}=15$. This result is of special significance given that single hole layers have now been fabricated having densities as low as $r_{s}=19$ [3].

The second layer not only affects the ground state properties but it also alters the dynamic properties of the liquid phase particularly near the phase transitions to the inhomogeneous ground states. For both the charge density wave and coupled Wigner crystal transitions we found development in the liquid of a soft mode that acts as a precursor for the transition to the corresponding inhomogeneous state. In the case of the transition to the electron solid we found evidence that the single-particle excitation spectrum was collapsing into a ridge connecting the plasmon dispersion curve and the soft mode centred at $q=G$ in such a way that the entire spectrum resembled the dispersion curve of the lowest lying phonon excitation for the electron solid. We interpret this as indicating that the change in the excitation spectrum of the liquid evolves continuously into the phonon line as the phase transition to the solid is approached.

\section{Acknowledgments}

We wish to thank to L. Liu for preparing the figures. One of us (L.S.) acknowledges support from the Australian Research Grants Fellowship program. 


\section{References}

[1] D.M. Ceperley, B.J. Alder, Phys. Rev. Lett. 45, 566 (1980).

[2] B. Tanatar, D.M. Ceperley, Phys. Rev. B 39, 5005 (1989).

[3] M.B. Santos, Y.W. Suen, M. Shayegan, Y.P. Li, L.W. Engel, D.C. Tsui, Phys. Rev. Lett. 68, 1188 (1992); M.B. Santos, J. Jo, Y.W. Suen, L.W. Engel, M. Shayegan, Phys. Rev. B 46, 13639 (1992).

[4] L. Świerkowski, D. Neilson, J. Szymański, Phys. Rev. Lett. 67, 240 (1991).

[5] D. Neilson, L. Świerkowski, J. Szymański, in: Strongly Correlated Electron Systems, Eds. D. Neilson, M.P. Das, Nova Science Publ., New York 1993, p.171.

[6] Yu.E. Lozovik, V.I. Yudson, Sov. Phys. JETP Lett. 22, 274 (1975); Solid State Commun. 19, 391 (1976); Sov. Phys. JETP 44, 389 (1976).

[7] S.I. Shevchenko, Sov. J. Low Temp. Phys. 2, 251 (1976).

[8] D. Neilson, L. Świerkowski, A. Sjölander, J. Szymaníski, Phys. Rev. B 44,6291 (1991).

[9] J. Hubbard, Phys. Lett. A 25, 709 (1967); Proc. R. Soc. Lond. A 243, 336 (1957).

[10] K.S. Singwi, M.P. Tosi, R.II. Land, A. Sjölander, Phys. Rev. 176, 589 (1968).

[11] A. Sjölander, J. Stott, Phys. Rev. B 5, 2109 (1972); P. Vashishta, P. Bhattacharyya, K.S. Singwi, Il Nuovo Cimento B 23, 172 (1974).

[12] K.S. Singwi, M.P. Tosi, in: Solid State Physics, Eds. H. Ehrenreich, F. Seitz, D. Turnbull, Vol. 36, Academic Press, New York 1981, p. 177.

[13] D. Neilson, L. Świerkowski, J. Szymański, in: Recent Progress in Many Body Theories, Eds. L. Blum, R.F. Bishop, Plenum, New York 1992.

[14] D.N. Lowy, A.D. Jackson, Phys. Rev. B 12, 1689 (1975).

[15] D. Belitz, S. Das Sarma, Phys. Rev. B 34, 8264 (1986).

[16] D. Neilson, L. Świerkowski, J. Szymański, L. Liu, J. Low Temp. Phys. 89, 251 (1992).

[17] L. Bonsall, A. Maradudin, Phys. Rev. B 15, 1959 (1977). 